\title{
Incidence of Acute Myocardial Infarction in Takashima, Shiga, Japan
}

\author{
Michiaki Yoshida, MD; Yoshikuni Kita, MD*; Yasuyuki Nakamura, MD**; \\ Akihiko Nozaki, MD*; Akira Okayama, $\mathrm{MD}^{\dagger}$; Hideki Sugihara, MD ${ }^{\dagger}$; \\ Takayuki Kasamatsu, MD $\$$; Kunihiko Hirose, MD $\$$; \\ Masahiko Kinoshita, MD; Hirotsugu Ueshima, MD*
}

\begin{abstract}
Background The incidence and mortality from ischemic heart disease (IHD) in Japan seem to be among the lowest of all the industrialized countries, but there are few reliable registers of acute myocardial infarction (AMI).

Methods and Results To assess the incidence of AMI in Takashima County, Shiga, Japan, from 1988 to 1998 and compare the data with similar registers in the world, cases of AMI or sudden death presumed from myocardial ischemia were registered. The criteria of AMI were based on the WHO MONICA Projects. The medical records of all the hospitals inside as well as outside the county, the original death records in the health center, and the ambulance records in the county were investigated and 291 cases were registered (190 males, 101 females; average age (mean $\pm \mathrm{SD}), 69.5 \pm 12.2$ ). The 28 -day and 24 -h case fatality was $38.1 \%$ and $33.0 \%$, respectively. Age-adjusted annual incidence of AMI per 100,000 population aged between 25 and 74 years were 58.2 for men and 18.0 for women. The incidence of AMI showed a constant trend from 1988 to 1998.
\end{abstract}

Conclusion The results confirmed that Japan has the lowest incidence of AMI among the industrialized countries. (Circ J 2005; 69: 404-408)

Key Words: Epidemiology; Japan; Myocardial infarction; Population

$\mathbf{T}$ he incidence and mortality from ischemic heart disease (IHD) in Japan seem to be among the lowest of all the industrialized countries and the ageadjusted IHD mortality in Japan has been decreasing gradually since approximately $19700^{1-4}$ However, there are few reliable community-based studies of acute myocardial infarction (AMI) in Japan ${ }^{5-9}$ and most of them included only a small number of annual events and had short research periods. The low mortality rates for IHD in Japan may be partly artifact, because the majority of deaths in the category of diseases of the heart are attributed to "heart failure" whereas this category is rarely used in other industrialized countries 10 Although the possibility that the mortality from IHD in the post World War II birth cohort in Japan might have actually increased has been suggested ${ }^{10}$ another study reported no increase at all11 Therefore, it is difficult to determine whether the incidence and mortality from AMI are increasing or decreasing in Japan.

Ongoing AMI registration was established in Takashima

(Received July 12, 2004; revised manuscript received January 5, 2005; accepted January 27, 2005)

Division of Cardiology, Department of Medicine, Shiga University of Medical Science, *Department of Health Science, Shiga University of Medical Science, Otsu, **Cardiovascular Epidemiology, Faculty of Home Economics, Kyoto Women's University, Kyoto, Department Preventive Cardiology, National Cardiovascular Center, Suita, tDepartment of Internal Medicine, Takashima General Hospital, \#akino Hospital, Takashima and $\$$ Department of Cardiology, Otsu Red Cross Hospital, Otsu, Japan

Mailing address: Yasuyuki Nakamura, MD, Cardiovascular Epidemiology, Department of Living and Welfare, Faculty of Home Economics, Kyoto Women's University, 35 Imakumano Kitahiyoshicho, Higashiyama-ku, Kyoto 650-8501, Japan. E-mail: nakamury@ kyoto-wu.ac.jp
County in Shiga Prefecture, Japan, in 1988 in order to measure trends in the incidence and case-fatality of AMI. At the same time, we have been registering stroke since $1988^{12}$ so that we can clarify the incidence ratio of stroke and AMI, and compare the incidence of AMI in Japan with other Western countries.

\section{Methods}

Geographic Conditions of the Research Area

Shiga prefecture is located in the approximate geographic center of Japan, bordering the Kyoto municipal area to the south-west. Lake Biwa, the largest lake in Japan, is located in the east of Takashima County and the Hira-san Gangs runs north-south to the west. Takashima County has a stable population of approximately 53,000 with $17.6 \%$ of the population (about 10,000 ) aged 65 years or older. It is a farming community, although only approximately $7 \%$ of the residents are engaged in primary industries; almost $38 \%$ of the residents are involved in secondary industries and 55\% in tertiary industries. The majority of residents in the latter 2 categories work outside the county.

There are 2 community hospitals in Takashima County; the one in the south is a public facility providing 261 beds, and the other in the north is a private facility providing 72 beds. Although percutaneous catheter interventional therapy for AMI ${ }^{13,14}$ had begun at public hospital in 1998, prior to this year almost all cases of AMI were transferred to tertiary hospitals (4 facilities) outside the county. However, almost all the patients in the county were taken to one of the 2 local hospitals by ambulance before transfer, so the Takashima hospitals had the medical records of the patients who were subsequently be registered as having AMI. 
Table 1 Fatal Cases of AMI in Takashima, Shiga, Japan, 1988-1998

\begin{tabular}{lc}
\hline \hline Total fatal cases within 28 days & 111 \\
Sudden cardiac death within $24 \mathrm{~h}$ & 96 \\
Out-of-hospital sudden death & 74 \\
Death within 24h or during transfer & 22 \\
Death from 24h to 28 days & 15 \\
28-day case fatality & $38.1 \%(111 / 291)$ \\
28-day case fatality for hospitalized cases & $17.1 \%(37 / 217)$ \\
28-day case fatality for hospitalized 24- $h$ survivors & $7.6 \%(15 / 195)$ \\
Pre-hospital case fatality & $25.4 \%(74 / 291)$ \\
24-h case fatality & $33.0 \%(96 / 291)$ \\
Pre-hospital death/all fatal cases & $66.7 \%(74 / 11)$ \\
Death within 24 h/all fatal cases & $86.5 \%(96 / 111)$ \\
\hline
\end{tabular}

AMI, acute myocardial infarction.

Table 2 Incidence of Case of Survival and Death After AMI by 10-Year Age Groups in Takashima, Shiga, Japan, 1988-1998

\begin{tabular}{|c|c|c|c|c|}
\hline $\begin{array}{l}\text { Age group } \\
\text { (years) }\end{array}$ & $\begin{array}{l}\text { Total cases } \\
\qquad(M / F)\end{array}$ & Non-fatal cases & $\begin{array}{c}\text { Fatal cases } \\
\text { after admission }\end{array}$ & $\begin{array}{c}\text { Fatal cases } \\
\text { before admission }\end{array}$ \\
\hline $20-29$ & $2(2 / 0)$ & $2(100.0 \%)$ & $0(0 \%)$ & $0(0 \%)$ \\
\hline $30-39$ & $3(2 / 1)$ & $2(66.7 \%)$ & $0(0 \%)$ & $1(33.3 \%)$ \\
\hline $40-49$ & $13(13 / 0)$ & $11(84.6 \%)$ & $1(7.7 \%)$ & $1(7.7 \%)$ \\
\hline $50-59$ & $35(29 / 6)$ & $23(65.7 \%)$ & $4(11.4 \%)$ & $8(22.9 \%)$ \\
\hline $60-69$ & $95(66 / 29)$ & $66(69.5 \%)$ & $5(5.3 \%)$ & $24(25.3 \%)$ \\
\hline $70-79$ & $79(49 / 30)$ & $49(62.3 \%)$ & $10(12.7 \%)$ & $20(25.3 \%)$ \\
\hline $80-89$ & $54(26 / 28)$ & $24(44.4 \%)$ & $15(27.8 \%)$ & $15(27.8 \%)$ \\
\hline$\geq 90$ & $10(3 / 7)$ & $3(30.0 \%)$ & $2(20.0 \%)$ & $5(50.0 \%)$ \\
\hline Total & $291(190 / 101)$ & $180(61.9 \%)$ & $37(12.7 \%)$ & $74(25.4 \%)$ \\
\hline
\end{tabular}

AMI, acute myocardial infarction.

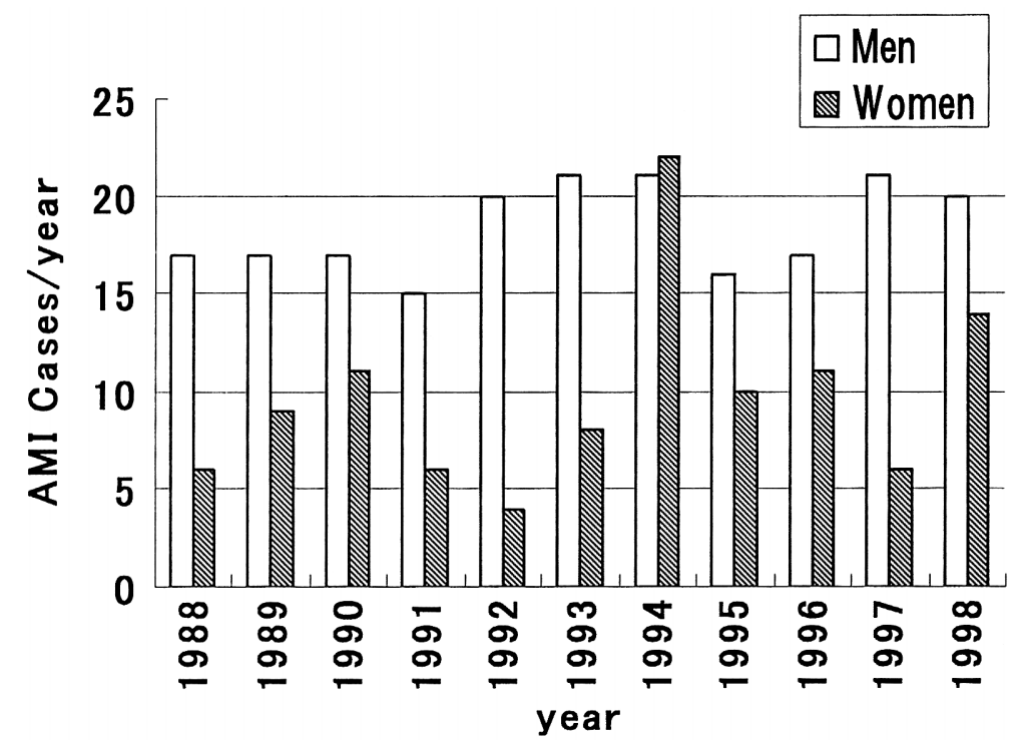

Fig 1. Annual incidence of AMI from 1988 to 1998 in Takashima, Shiga, Japan. There is a constant trend from 1988 to 1998, except for 1994, but the difference is not statistically significant by chi-square test.

\section{Registration}

Registered patients included all those who were residents of Takashima County and had either experienced an AMI died out-of-hospital from sudden cardiac death suspected to be related to myocardial ischemia. The criteria of AMI were based on the WHO MONICA Projects (Monitoring of Trends and Determinants in Cardiovascular Disease) 15 We registered all cases that met the inclusion criteria on the basis of the medical records from all the relevant hospitals inside and outside the county. We investigated the original death records at the county health center and the county ambulance records with the permission of the Ministry of
Public Management, Home Affaires, Post and Telecommunications, Japan. Patients' privacy was protected.

We used the registration form of the Monitoring System for Cardiovascular Disease commissioned by the Ministry of Health and Welfare ${ }^{16}$ Items recorded at registration of an AMI were the date and time of onset, age, sex, location at the time of onset, time of admission, electrocardiographic findings, region of infarction, Q wave or non-Q-wave myocardial infarction (MI), peak creatine kinase, coronary risk factors, history of MI, pre-infarction angina, complications in the acute stage, Killip's classification, fatality within 28 days, cause of death, angiographic findings, acute therapy, 
Men

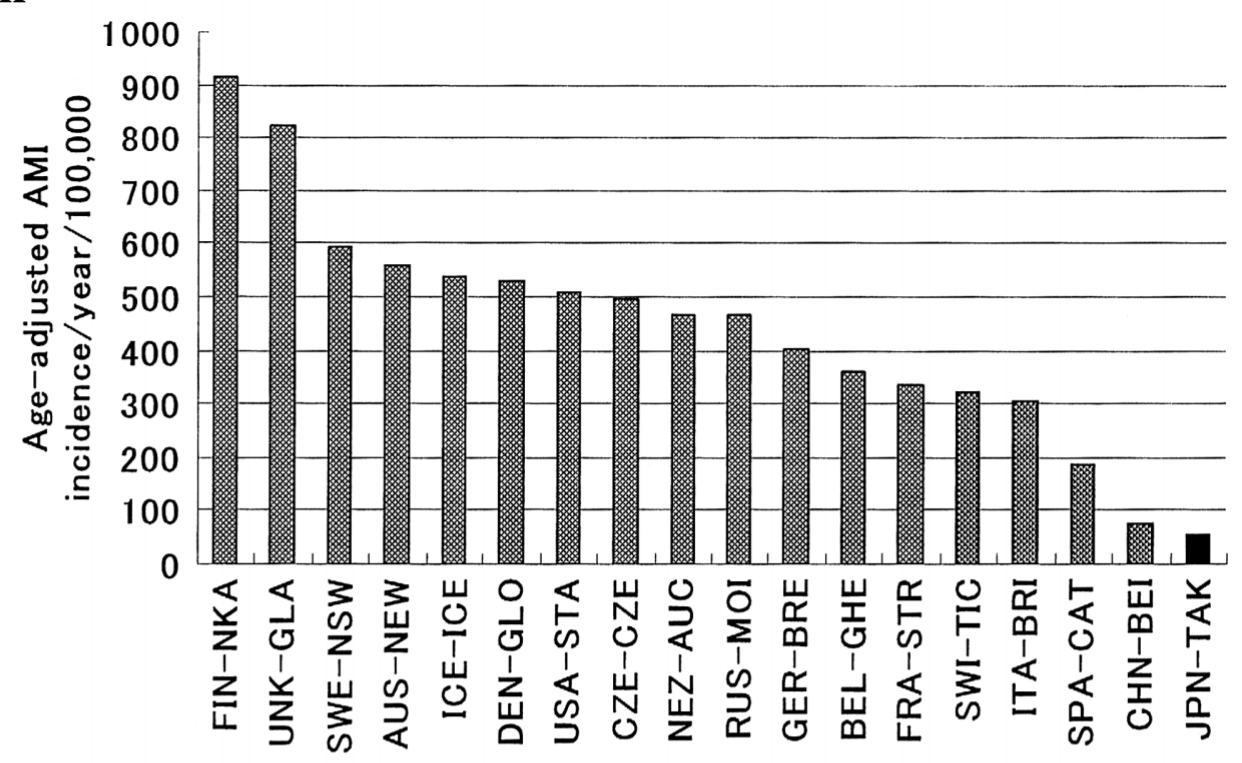

Population

\section{Women}

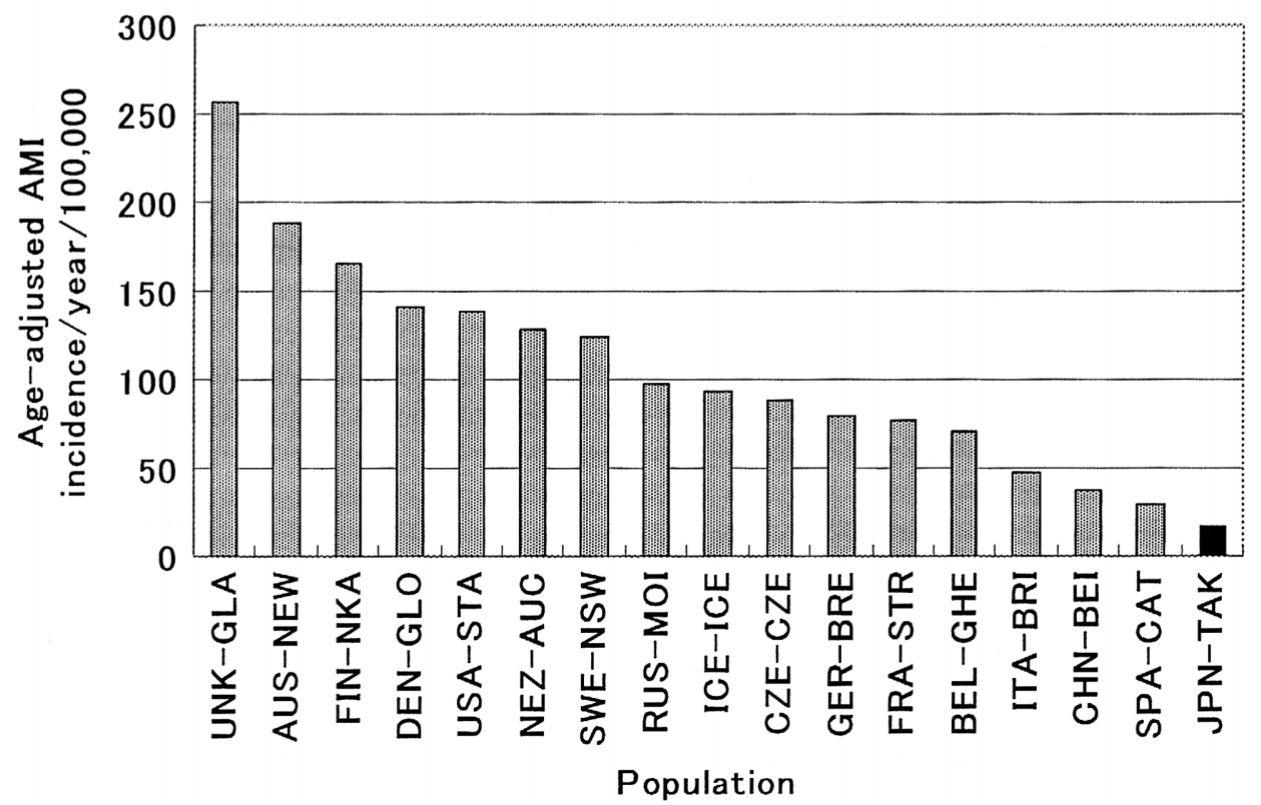

Fig 2. Age-adjusted annual incidence rate of first AMI per 100,000 population for men (Top) and women (Bottom) aged between 35 and 64 years compared with WHO MONICA Project (WHO MONICA data 1985-1987). The incidence rates of AMI in Takashima County in 1988-1998 were the lowest in the world for both men and women. FIN-NKA, Finland-North Karelia; UNK-GLA, United Kingdom-Glasgow; ICE-ICE, Iceland-Iceland; AUS-NEW = AustraliaNewcastle; SWE-NSW, Sweden-Northern Sweden; DEN-GLO, Denmark-Glostrup; NEZ-AUC, New Zealand-Auckland; USA-STA, United States of America-Stanford; CZE-CZE, Czech Republic-Czech Republic; BEL-GHE, Belgium-Ghent; GER-BRE, Germany-Bremen; FRA-STR, France-Strasbourg; RUS-MOI, Russia-Moscow Intervention; ITA-BRI, ItalyArea Brianza; SWI-TIC, Switzerland-Ticino; SPA-CAT, Spain-Catalonia; CHN-BEI, China-Beijing; JPN-TAK, JapanTakashima.

and New York Heart Association functional classification ${ }^{17}$ at discharge.

Definite sudden cardiac death was defined as fatal cases with gross evidence of fresh myocardial infarction and/or recent coronary occlusion at necropsy. Possible sudden cardiac death was defined as fatal cases in which there was no other strong evidence of another cause of death, clinically or at autopsy, with typical, atypical or inadequately described symptoms; or without typical, atypical or inadequately described symptoms but with evidence of chronic coronary occlusion or stenosis or old myocardial scarring at necropsy; or a history of chronic IHD such as definite or possible MI, coronary insufficiency or angina pectoris in the absence of significant valvular disease or cardiomyopathy.

In cases of silent MI diagnosed later, the onset dates 
were taken as the oldest possible date of documentation.

\section{Data Analysis}

We calculated age-specific rates in 10-year age groups and age-specific incidence rates were determined by the population of the relevant age group in Takashima County at the 1993 census ${ }^{18}$ We calculated 2 types of age-adjusted incidence rates, using the Japanese population aged 20 years and older, and those aged between 35 and 64 years from the 1980 census as the standard population to allow comparison with the results of the WHO MONICA Project 19 Direct age standardization of the incidence rates was done according to the WHO MONICA Project, using the truncated Segi world standard population weights $6,6,6,5,4$ and 4 for the 5-year age groups of 35-39 to 60-64 years, respectively. 20

\section{Results}

We registered 291 cases (males: 190 cases; females: 101 cases; averaged age (mean $\pm S D)$ : 69.5 \pm 12.2 , max: 96 years; min: 29 years) during 11 years between the beginning of 1988 and the end of 1998, which included sudden cardiac death cases caused by definite or possible myocardial ischemia. Table 1 shows the fatal cases. The total number of fatal cases within 28 days was 111 (approximately $2.4 \%$ of the total mortality of all the ages during the 11 years), which included 74 cases of out-of-hospital sudden cardiac death or cardiopulmonary arrest, 22 cases of sudden cardiac death within $24 \mathrm{~h}$ of arriving at hospital or during transfer to tertiary hospital, and 15 cases of death from $24 \mathrm{~h}$ to 28 days; in total there were 96 cases of sudden cardiac death within $24 \mathrm{~h}$ and 37 cases of death after admission and within 28 days. The 28-day case fatality, 28-day case fatality for hospitalized cases and 28-day case fatality for hospitalized 24-h survivors was $38.1 \%$ (111/291), $17.1 \%(37 / 217)$ and $7.6 \%(15 / 195)$, respectively. Prehospital case fatality and 24-h case fatality was $25.4 \%$ (74/291) and 33.0\% (96/291), respectively. The rate of prehospital death for all fatal cases were $66.7 \%$ (74/111) and the rate of death within $24 \mathrm{~h}$ for all fatal cases was $86.5 \%$ (96/111).

Table 2 shows the incidence of survival and fatal cases by 10 -year age groups. The peak incidence by 10 -year age groups was in the 60-69 year olds. The ratio of males to females was very high in the age groups under 59 years, but the percentage of females became higher with advancing age and the ratio was less than one in the groups over 80 years old. There was a tendency for the total case fatality to became higher with advancing age. The incidence of AMI showed a constant trend from 1988 to 1998 except for the year 1994 (Fig 1).

Table 3 shows the age-specific and age-adjusted annual incidence rate of first AMI per 100,000 population aged 20 years and older. The age-specific incidence rates for the total and for men and women, respectively, increased with advancing age.

Age-adjusted incidence rates for those aged between 35 and 64 years were 55.5/year per 100,000 population for men and 9.1/year per 100,000 population for women. The comparison of the age-adjusted annual incidence rates for men and women aged from 35 to 64 years with the WHO MONICA Project ${ }^{15}$ is shown in Fig 2.
Table 3 Age-Specific and Age-Adjusted Annual Incidence Rates of First AMI per 100,000 Population Aged 20 Years and Older in Takashima, Shiga, Japan, 1988-1998

\begin{tabular}{lrrr}
\hline \hline Age group (years) & Total & Male & Female \\
\hline $20-29$ & 3.0 & 6.0 & 0 \\
$30-39$ & 4.3 & 5.7 & 2.8 \\
$40-49$ & 13.8 & 24.2 & 0 \\
$50-59$ & 47.5 & 80.0 & 16.4 \\
$60-69$ & 109.9 & 155.9 & 66.8 \\
$70-79$ & 152.8 & 239.5 & 96.9 \\
$\geq 80$ & 258.0 & 346.8 & 210.8 \\
Crude rate & 61.5 & 83.5 & 41.1 \\
Age-adjusted ratea & 42.3 & 65.2 & 23.0 \\
\hline
\end{tabular}

Adjusted to 1980 Japanese population aged 20 years and older.

\section{Discussion}

We found that the incidence of AMI in Takashima county, Japan showed a constant trend from 1988 to 1998 except for 1994, and our results confirmed that Japan has the lowest incidence of AMI among the industrialized countries. A comprehensive registration system for diseases such as AMI is sine qua non in determining the incidence in a particular area. A system to capture all patients in the study area, together with accurate diagnosis, is required to ensure comprehensive registration. Factors that reduce the comprehensiveness of a register are missing cases of sudden death for which there is not a confirmed diagnosis, missing AMI patients admitted to hospitals outside the registration area, and non-registration because of the AMI patient being cared for at home or in a nursing home.

We registered cases of out-of-hospital sudden cardiac death, but because electrocardiogram findings and the concentrations of cardiac enzymes are often not available in such cases, we had to base registration on the patients' location and symptoms at onset and their history of coronary heart disease. We tried to register only cases with definite and possible causes of sudden coronary death according to the MONICA registry's definition. The rate of pre-hospital death for all fatal cases was $66.7 \%$ in this study. In the WHO MONICA Project, the average rates for men and women were $70 \%$ and $64 \%$, respectively. ${ }^{21}$ Therefore, we believe that cases of other causes of death were excluded from registration as cases of sudden cardiac death in this study.

To ensure that eligible patients hospitalized outside the county were not excluded, registration was also conducted at the main tertiary hospitals. For example, 23 cases were not transferred to either of the local hospitals in the acute phase, but 12 had county ambulance records and we obtained information of the other 11 cases from the hospitals outside the county: of these, 8 in-hospital deaths were located in the original death records in the county health center ( 5 occurred outside the prefecture, 3 within the prefecture) and the remaining 3 cases were hospitalized outside the county without using the county ambulance system. The major limitation of our data collection related to cases of non-fatal AMI managed outside the 4 main medical facilities; however, we estimated that there would be very few patients who would go to hospitals that were not participating in our registration system.

Almost $100 \%$ of the residents of Japan have public health insurance offered by sources such as the Ministry of Health and Welfare. Health insurance is not expensive and 
the policies cover all diseases, excluding injury from road traffic accident. Therefore, the usual practice in Japan is that patients in the county would consult a general physician, who if AMI was suspected from the symptoms and signs would almost always refer the patient to a secondary or tertiary hospitals for extensive investigation. Therefore we believe that only a few cases of non-fatal AMI were not registered.

We have conducted a previous registration of AMI cases in 7 areas in Japan, including Takashima County, based mainly on hospital records. The age-adjusted first AMI incidence rates per 100,000 for all ages in Akita, Ehime, Nagano, Takashima, Hokkaido, Osaka and Okinawa areas were $15.0,19.5,22.6,22.6,24.7,24.9$, and 26.0 respectively.2 Therefore, the incidence rates in Takashima in the median of the 7 regions. In the present study, we extended the comprehensiveness of the register by investigating the original death records at the county health center and the ambulance records; as a result, the first AMI incidence rate increased from 22.6 per 100,000 population of all ages to 30.5 .

Although we have registered cases of AMI and stroke in this area since 1988, the incidence of AMI showed a constant trend from 1988 to 1998 except for the year 1994 (Fig 1). Some Japanese studies have reported that the incidence of AMI did not vary much during the research period 5,7 and although many cardiologists and physicians are under the impression that the incidence of AMI in Japan is increasing, the crude incidence has not changed greatly in a decade. Before 1993, many physicians in Japan used the term "acute heart failure" for sudden death of unknown etiology for the diagnosis on the medical certificate, but in 1994 the Ministry of Health and Welfare recommended that it not be used; however, some physicians might have used the term "AMI", even though they were not certain of the definite diagnosis and this may be a reason for the higher incidence of AMI in women in 1994 than in the other years. As the incidence of AMI in men did not change in 1994, it is likely that the increased incidence in women in 1994 was a random fluctuation.

The age-adjusted annual incidence rates for men and women aged from 35 to 64 years were compared with those of the WHO MONICA Project ${ }^{15}$ (Fig 2). Takashima County has the lowest incidence in the world for AMI in men, women and both sexes. Our results confirmed that Japan has the lowest incidence of AMI among the industrialized countries as reported by other investigators. Ongoing monitoring of AMI in Takashima County is feasible and will promote understanding of the reasons for the continuing decline in AMI incidence that has been noted in all regions of Japan.

\section{Acknowledgments}

This study was supported in part by grants (3A-1, 6A-5 and 7A-2) from The Research on Cardiovascular Disease of Ministry of Health and Welfare, and C-2 13670361 from the Grants-in-Aid Scientific Research on Ministry of Education, Culture, Sports, Science and Technology.

\section{References}

1. Uemura K, Pisa Z. Recent trends in cardiovascular disease mortality in 27 industrialized countries. World Health Stat $Q 1985 ; 38$ : $142-$ 162 .
2. Uemura K, Pisa Z. Trends in cardiovascular disease mortality in industrialized countries since 1950. World Health Stat $Q$ 1988; 41: $155-178$.

3. Hatano S. Changing CHD mortality and its causes in Japan during 1955 - 1985. Int J Epidemiol 1989; 18: S149-S158.

4. Ueshima H, Tatara K, Asakura S. Declining mortality from ischemic heart disease and changes in coronary risk factors in Japan, 19561980. Am J Epidemiol 1987; 125: 62-72.

5. Kodama K, Sasaki H, Shimizu Y. Trend of coronary heart disease and its relationship to risk factors in a Japanese population: A 26-year follow up, Hiroshima/Nagasaki study. Jpn Circ J 1990; 54: 414421.

6. Kinjo K, Kimura Y, Shinzato Y, Tomori M, Komine Y, Kawazoe N, et al. An epidemiological analysis of cardiovascular disease in Okinawa, Japan. Hypertens Res 1992; 15: 111-119.

7. Shimamoto T, Komachi Y, Inada H, Doi M, Iso H, Sato S, et al. Trends for coronary heart disease and stroke and their risk factors in Japan. Circulation 1989; 79: 503-515.

8. Konishi M, Iida M, Naito Y, Terao A, Takayama Y, Ito H, et al. The trend of coronary heart disease and its risk factors based on epidemiological investigation. Jpn Circ J 1987; 51: 319-324.

9. Shimozato M, Nakayama T, Yokoyama T, Yoshi-ike N, Yamaguchi M, Date C. A 15.5-year cohort study on risk factors for possible myocardial infarction and sudden death within 24 hours in a rural Japanese community. J Epidemiol 1996; 6: 15-22.

10. Sekikawa A, Kuller LH, Ueshima H, Park JE, Suh I, Jee SH, et al. Coronary heart disease mortality trends in men in the post World War II birth cohorts aged 35-44 in Japan, South Korea and Taiwan compared with the United States. Int J Epidemiol 1999; 28: $1044-$ 1049.

11. Okayama A, Ueshima H, Marmot M, Elliott P, Choudhury SR, Kita $Y$. Generational and regional differences in trends of mortality from ischemic heart disease in Japan from 1969 to 1992. Am J Epidemiol 2001; 153: $1191-1198$.

12. Kita Y, Okayama A, Ueshima H, Wada M, Nozaki A, Choudhury SR, et al. Stroke incidence and case fatality in Shiga, Japan 1989-1993. Int J Epidemiol 1999; 28: 1059-1065.

13. Nishigaki K, Yamazaki T, Fukunishi M, Tanihata S, Fujiwara H, Japanese Coronary Intervention Study Group. Assessment of acute myocardial infarction in Japan by the Japanese Coronary Intervention Study (JCIS) Group. Circ J 2004; 68: 515-519.

14. Shihara M, Tsutsui H, Tsuchihashi M, Shigematsu H, Yamamoto S, Koike G, et al Japanese Coronary Intervention Study (JCIS) Group. Coronary revascularization in Japan. Part 3: Percutaneous coronary intervention during 1997. Circ J 2002; 66: 10-19.

15. Tunstall-Pedoe H, Kuulasmmaa K, Amouyel P, Arveiler D, Rajakangas AM, Pajak A. Myocardial infarction and coronary deaths in the World Health Organization MONICA Project: Registration procedures, event rates, and case-fatality rates in 38 populations from 21 countries in four continents. Circulation 1994; 90: 583-612.

16. Ministry of Health and Welfare. Study Project of Monitoring System for Cardiovascular Disease commissioned by the Ministry of Health and Welfare: Manual for the Registry and Follow-up of Ischemic Heart Disease. Osaka: National Cardiovascular Center, 1998 (in Japanese).

17. The Criteria Committee of the New York Heart Association. Nomenclature and Criteria for Diagnosis, 9th edn. Boston: Little Brown; 1994.

18. Shiga Prefectual Imazu Public Health Center. 1993 Annual Report of Imazu Public Health Center. Shiga Prefectual Imazu Public Health Center, Takashima, Shiga, Japan, 1991 (in Japanese).

19. Statistics and Information Department, Minister's Secretariat, Ministry of Health Welfare. About age-adjusted death rate. Jpn J Public Health 1991; 38: 535.

20. Waterhouse J, Muir C, Correa P, editors. Cancer incidence in five continents, Vol. III. IARC Scientific Publication. Lyon, France: International Agency for Research on Cancer, 1976.

21. Chambless L, Keil U, Dobson A, Mahonen M, Kuulasmaa K, Rajakangas AM, et al. Population versus clinical view of case fatality from acute coronary heart disease: Results from the WHO MONICA Project 1985-1990. Circulation 1997; 96: 3849-3859.

22. Isomura K. Studies on the development of long-term follow-up system of cardiovascular diseases: Community-based task. In: Annual Report of the Research on Cardiovascular Diseases 1993. Tokyo: National Cardiovascular Center; 1993; 19-21. 